\title{
Performance of Sri Lankan FRAX algorithm without bone mineral density and with Quantitative Ultrasound data input
}

\author{
H W A S Subasinghe ${ }^{1}$, S Lekamwasam ${ }^{2}$, P Ball ${ }^{3}$, H Morrissey $^{3}$, E I Waidyaratne ${ }^{4}$
}

(Index words: Bone mineral density, FRAX, Postmenopausal women, Ultrasound bone scan)

\begin{abstract}
Introduction Fracture risk assessment algorithm (FRAX) is the most validated method available to predict fracture risk. Its use is restricted due to limited availability of Dual Energy X-ray Absorptiometry (DXA). FRAX has the option of assessing fracture risk without bone mineral density (BMD) data.

Objectives To assess the ability of Sri Lankan FRAX algorithm without BMD input in evaluating fracture risk. The possibility of replacing the BMD input with Quantitative Ultrasound (QUS) data of radius in calculating fracture risk also assessed.

Methods Data of clinical risk factors associated with fractures were collected from community dwelling postmenopausal women $(n=339)$. DXA scans were performed in all subjects and QUS scans (in radius) were performed in a randomly selected sample $(n=207)$. Ten-year risks of major osteoporotic fracture (MOFR) and hip fracture (HFR) were calculated with BMD, without BMD (FRAX-FN ${ }_{0}$ ) and with QUS T score instead of BMD (FRAX-UST).
\end{abstract}

Results and conclusion Nearly $35.7 \%$ had high risk of fractures. FRAX-FN $\mathrm{FN}_{0}$ had $79.2 \%$ sensitivity, $80.1 \%$ specificity, $68.8 \%$ positive predictive value (PPV) and $87.4 \%$ negative predictive value (NPV). FRAX-UST showed $78.4 \%$ sensitivity, $70 \%$ specificity, $59.8 \%$ PPV and $85 \%$ NPV. ROC AUCs were above 0.80 in both FRAX$\mathrm{FN}_{0}$ and FRAX-UST. The standard errors of estimate (SEE) were less in FRAX-FN 0 (3.96 and 2.76 for MOFR$\mathrm{FN}_{0}$ and HFR-FN $\mathrm{N}_{0}$ respectively) compared to FRAX-UST (6.13 and 4.83 for MOFR-UST and HFR-UST, respectively). In conclusion, Sri Lankan FRAX without BMD is an acceptable alternative in areas with restricted DXA facility. Radial QUS data cannot be used as a substitute to FN-BMD in Sri Lankan FRAX.

Ceylon Medical Journal 2019; 64: 17-24

DOI: http://doi.org//10.4038/cmj.v64i1.8836

\section{Introduction}

Osteoporosis which is characterized by low bone mineral density (BMD) and microarchitectural deterioration is associated with increased fracture risk especially in elderly [1,2]. According to the International Osteoporosis Foundation, osteoporosis leads to more than 8.9 million fractures worldwide, annually, and every 1 in 3 women and 1 in 5 men are at risk of osteoporotic fracture, during the lifetime [3]. Fracture incidence varies in different populations and the rates are expected to increase continuously as the global population turn older. The USA experienced two million osteoporotic fractures in 2005 and this figure is projected to reach three million in 2025 [4,5]. Elderly population in Asia is rapidly expanding and in $2050,50 \%$ of total hip fractures would be seen in the Asian region [6-8].

In order to reduce osteoporotic fractures, high risk patients need to be detected and intervened early. Though there are many tools available to assess fracture risk, FRAX (Fracture Risk Assessment Tool), is the most validated and widely used. It has been developed based on clinical risk factors which have proven associations with low bone density or occurrence of fractures by large epidemiological studies [9]. FRAX algorithm which can be applied to both men and women between 40-90 years estimates the 10-year probability of both hip and major osteoporotic fractures [9].

Despite the wide use, FRAX has major limitations. FRAX needs femoral neck (FN) BMD assessed by either Dual Energy X-ray Absorptiometry (DXA) or Quantitative Computed Tomography (QCT) as an input variable thus restricting its full application in countries with limited facilities. As an alternative, attempts have been made to estimate fracture risk without BMD data. Some studies have shown that the forecast of 10-year fracture probability with and without BMD is comparable [10-12]. However, some studies have shown conflicting results [13-15]. It is intuitive to think that the non-inclusion of BMD is

${ }^{1}$ Department of Pharmacy, Faculty of Allied Health Sciences, University of Ruhuna, ${ }^{2}$ Department of Medicine, Faculty of Medicine, University of Ruhuna, ${ }^{3}$ School of Pharmacy, University of Wolverhampton, United Kingdom, ${ }^{4}$ Department of Anatomy, Faculty of Medicine, University of Ruhuna, Sri Lanka.

Correspondence: LK, e-mail: <sewwandisubasinghe@gmail.com>. Received 19 December 2018 and revised version accepted 06 March 2019.

This is an open-access article distributed under the terms of the Creative Commons Attribution License, which permits unrestricted use, distribution, and reproduction in any medium, provided the original author and source are credited. 
compensated, at least partly, by inclusion of other clinical risk factors (CRF) which are independent determinants of BMD. However, the ability of CRFs to make this compensation is unclear.

Though DXA is the gold standard in assessing BMD [16-18] quantitative ultrasound (QUS) is used to detect bone strength and fracture risk, particularly in less resourceful settings $[19,20]$. QUS is cheaper, portable and free of ionized radiation, hence can be used in many situations [21,22]. QUS does not measure BMD but it captures bone texture and generates measures such as Speed of Sound (SOS) and Broadband Ultrasound Attenuation (BUA). If QUS data, especially T-Score, can be used instead of femoral neck BMD in assessing fracture risk, it can partly overcome the restricted use of FRAX owing to the lack of DXA facility in many countries. However, we were unable to find studies examining the possibility of replacing BMD with QUS data in FRAX.

The objective of this study was to assess the impact of non-inclusion of femoral neck BMD on the fracture risk evaluation based on Sri Lankan FRAX algorithm. Further, we assessed the possibility of replacing the femoral neck BMD input with US data in calculating fracture risk.

\section{Materials and methods}

This cross-sectional study was conducted in Galle district in southern Sri Lanka. Ethical approval was obtained from the Ethical Review Committee of Faculty of Medicine, University of Ruhuna prior to data collection (Ref No. 09.03.2016:3.3).

Three Pradeshiya Sabha Divisions (intermediate administrative unit) were selected from Galle district in random manner. Two Gramasewa divisions (the smallest administrative unit) from each Pradeshiya Sabha division were selected, randomly, for data collection. Posters were displayed in public places of selected Gramasewa divisions to inform the public about the research. Community-dwelling postmenopausal women who voluntarily filled the 'expression of interest form' were invited to take part in the study. Women with chronic diseases of liver, kidney, heart, lungs or endocrine organs were excluded. Furthermore, women on medications that could affect vitamin D metabolism (hormonal contraceptives, diuretics, vitamin D supplementations) were also excluded from the study sample.

All participants $(n=339)$ were educated about the research and their written consent was obtained before enrolling into the study. Data were collected by interviewing them individually using a content validated datasheet. Weight and height were measured adhering to the standard protocols. Ultrasound bone scan (Sunlight Mini Omni, Israel) of the radius of the non-dominant side was performed in 207 randomly selected participants. All US scans were done by one trained investigator adhering to manufacturer's protocol and the machine was calibrated on each day prior to scanning. BMDs of the total spine (L1-L4), femoral neck and total hip were measured with DXA scan (Hologic Discovery W, Hologic Inc, Bedford, MA, USA). All the scans were performed by a trained technician adhering to manufacturer's protocol and the machine was calibrated on each day prior to scanning. Women were categorized as osteoporosis, osteopenia or normal, based on the $\mathrm{T}$ scores adhering to the WHO criteria [23,24].

Both major osteoporotic fracture risk (MOFR) and hip fracture risk (HFR) were calculated using the Sri Lankan FRAX tool (SL-FRAX). Calculations were modified and done twice; (i) without femoral neck BMD (FRAX-FN ${ }_{0}$, (ii) and with US T-score in place of femoral neck T-score (FRAX-UST).

The DXA scanner generated FRAX values which included femoral neck BMD (FRAX-A) were considered the reference standard. MOFR $\geq 11 \%$ and $\mathrm{HFR} \geq 3 \%$ were taken as intervention thresholds (25) and participants were categorized to high or low risk based on these cut-off values.

\section{Statistical Analysis}

MOFR and HFR of modified methods were compared with MOFR-A and HFR-A, respectively by paired t-test to assess differences. We also assessed the sensitivity, specificity, PPV and NPV of modified FRAX tools with regards to their ability to discriminate between high and low risk women compared to FRAX-A. Bland-Altman plots were constructed to determine the agreement between estimated and actual fracture probabilities. Receiver operating characteristic (ROC) curves were drawn and area under the curves (AUC) were determined in order to assess the fracture risk prediction of the new models based on actual fracture risk categorization by FRAX-A. Further, regression analyses were conducted for each modified FRAX tool to estimate how they predicted FRAX-A values. Standard Error of the Estimate (SEE) was considered in assessing the goodness of the fit and the accuracy of the predictions.

\section{Results}

The study sample included 339 postmenopausal women and their descriptive data are shown in Table 1. The prevalence of osteoporosis was $74.3 \%$ in the study sample and another $21.5 \%$ had osteopenia.

Table 2 shows the mean differences between the FRAX-A and modified FRAX outputs. MOFR-FN ${ }_{0}$ and HFR-FN ${ }_{0}$ showed the lowest mean differences when compared with MOFR-A and HFR-A, respectively. In contrast, MOFR-UST and HFR-UST showed comparatively higher mean differences. 
Table 1. Descriptive characteristics of the study sample

\begin{tabular}{lrr}
\hline Variable & Mean $(S D) / n(\%)$ & \multicolumn{1}{c}{ Range } \\
\hline Age $($ years $)$ & $63.8(9.3)$ & $40-87$ \\
Body weight $(\mathrm{kg})$ & $51.7(0.4)$ & $24.0-85.8$ \\
Height $(\mathrm{cm})$ & $147.8(5.6)$ & $130.0-163.0$ \\
BMI $\left(\mathrm{kg} / \mathrm{m}^{2}\right)$ & $23.6(4.4)$ & $12.7-37.7$ \\
Femoral neck BMD $\left(\mathrm{g} / \mathrm{cm}^{2}\right)$ & $0.634(0.129)$ & $3475-4790$ \\
Ultrasound SOS $(\mathrm{m} / \mathrm{s})$ & $3946(189)$ & \\
Previous fracture & $53(15.6 \%)$ & \\
Parent fractured hip & $14(4.1 \%)$ & \\
Current smoking & 0 & \\
Glucocorticoids use & $76(22.4 \%)$ & \\
Rheumatoid arthritis & $69(20.3 \%)$ & \\
Secondary osteoporosis & $6(1.8 \%)$ & \\
Alcohol 3 or more units/day & 0 & \\
\hline
\end{tabular}

Table 2. Comparison of modified FRAX outputs with FRAX-A

\begin{tabular}{|c|c|c|c|c|c|}
\hline & \multirow[t]{2}{*}{ Pair of variables compared } & \multirow[t]{2}{*}{$\begin{array}{c}\text { Mean } \\
\text { difference }\end{array}$} & \multirow[t]{2}{*}{$S D$} & \multicolumn{2}{|c|}{$\begin{array}{l}95 \% \text { Confidence Interval of } \\
\text { the mean difference }\end{array}$} \\
\hline & & & & Lower & Upper \\
\hline Pair 1 & MOFR-A- MOFR-FN0 & -0.11 & 5.63 & -0.72 & 0.49 \\
\hline Pair 2 & MOFR-A- MOFR-UST & -4.86 & 12.39 & -6.57 & -3.15 \\
\hline Pair 3 & HFR-A- HFR-FNO & -0.37 & 4.98 & -0.90 & 0.16 \\
\hline Pair 4 & HFR-A - HFR-UST & -4.22 & 11.69 & -5.83 & -2.60 \\
\hline
\end{tabular}

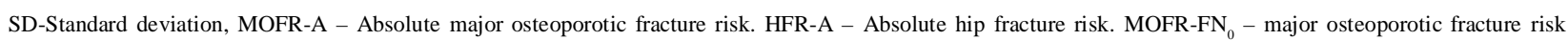
calculated without bone mineral density. HFR-FN ${ }_{0}$ - hip fracture risk calculated without bone mineral density. MOFR-UST - major osteoporotic fracture risk calculated with ultrasound T score. HFR-UST- hip fracture risk calculated with ultrasound T score.

In the assessment of fracture risk, FRAX-A detected $35.7 \%$ of study participants to be in the high-risk category based on the intervention thresholds adopted while FRAX-FN ${ }_{0}$ showed $41 \%$ to have a high fracture risk. The percentage of females categorised into low and high risk groups based on the performance of each FRAX model is given in table 3 .

Table 3. Ability of FRAX tools to identify high fracture risk

\begin{tabular}{cllcc}
\hline FRAX tool used & $\begin{array}{c}\text { 10-year fracture } \\
\text { probability }\end{array}$ & $\begin{array}{c}\text { Intervention } \\
\text { threshold score }\end{array}$ & $\begin{array}{c}\text { \% at high risk of } \\
\text { fractures }\end{array}$ & $\begin{array}{c}\text { \% of women } \\
\text { needed treatments }\end{array}$ \\
\hline \multirow{2}{*}{ FRAX-A } & MOFR-A & High risk $\geq 11$ & 26.8 & 35.7 \\
& HFR-A & High risk $\geq 3$ & 35.7 & 41 \\
FRAX-FN & MOFR-FN $_{0}$ & High risk $\geq 11$ & 28.6 & 47.8 \\
& HFR-FN & High risk $\geq 3$ & 41 & \\
FRAX-UST & MOFR-UST & High risk $\geq 11$ & 42.5 & 46.9 \\
& HF-UST & High risk $\geq 3$ & 4 \\
\hline
\end{tabular}

FRAX-A - Absolute Fracture risk assessment tool, MOFR-A - Absolute major osteoporotic fracture risk. HFR-A - Absolute hip fracture risk. FRAX-FN - FRAX without bone mineral density, MOFR-FN $\mathrm{N}_{0}$ - major osteoporotic fracture risk calculated without bone mineral density. HFR-FN $\mathrm{N}_{0}$ - hip fracture risk calculated without bone mineral density. FRAX-UST- FRAX with ultrasound T score, MOFR-UST - major osteoporotic fracture risk calculated with ultrasound T score. HFR-UST- hip fracture risk calculated with ultrasound $\mathrm{T}$ score. 
FRAX-FN ${ }_{0}$ showed high sensitivity (79.2\%) and specificity $(80.1 \%)$ in detecting women with high fracture risk. Further, PPV and NPV of FRAX-FN ${ }_{0}$ were $68.8 \%$ and $87.4 \%$, respectively. Though, FRAX-UST had high sensitivity (78.4\%), its specificity was low (70\%) indicating high possibility of misclassifying fracture risk. Furthermore, FRAX-UST had low PPV (59.8\%) although its NPV was $85 \%$.

Bland-Altman plots (Figures 1-4) showed the agreement between the actual and estimated fracture risk probabilities. MOFR-FN ${ }_{0}$ and HFR-FN ${ }_{0}$ showed good agreement with respective actual values. The agreement

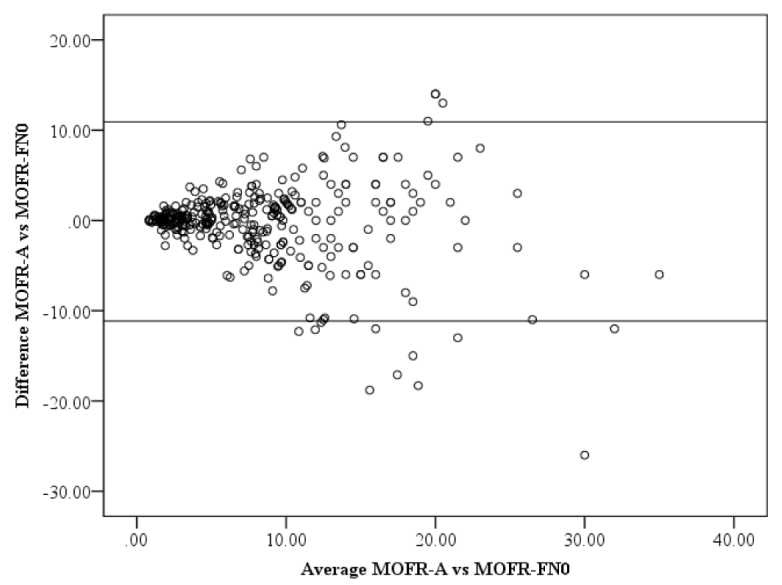

Figure 1. Bland Altman plot of MOFR-A and MOFR-FN

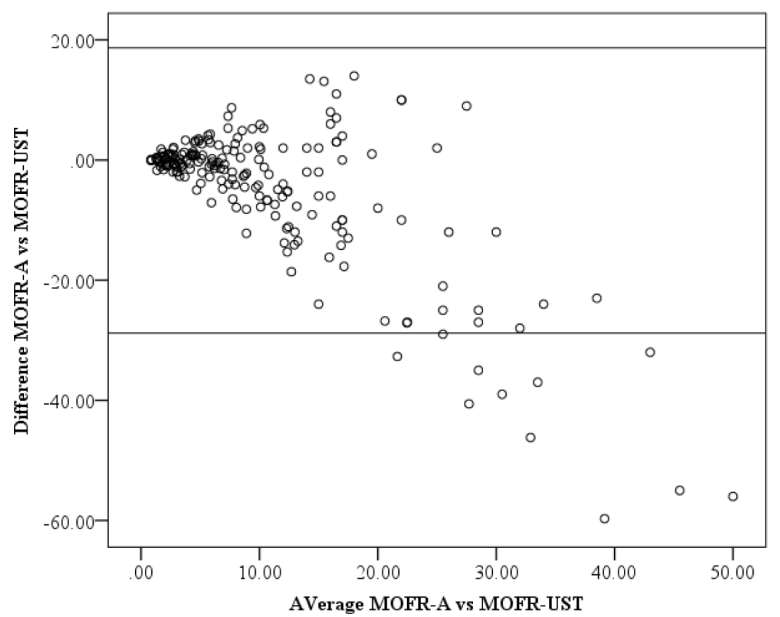

Figure 3. Bland Altman plot of MOFR-A and MOFR-UST. of MOFR-UST and HFR-UST with the respective actual values were lost at higher estimated values.

Figures 5 and 6 show the ROC curves for FRAX-FN and FRAX-UST, respectively. AUC of MOFR-FN0 was 0.88 (95\% CI 0.85 to $0.92, \mathrm{p}<0.001)$ and HFR-FN0 was 0.89 (95\% CI 0.85 to $0.92, \mathrm{p}<0.001$ ). The corresponding values for MOFR-UST and HFR-UST were 0.85 (95\% CI 0.79 to $0.89, \mathrm{p}<0.001)$ and $0.83 \mathrm{AUC}(95 \% \mathrm{CI} 0.77$ to $0.88, \mathrm{p}<0.001)$ respectively. Comparison showed that AUCs of FRAX$\mathrm{FN}_{0}(0.88$ of MOFR and 0.89 of HFR) were significantly higher $(\mathrm{p}<0.001)$ than those of FRAX-UST $(0.85$ of MOFR and 0.83 of HFR).

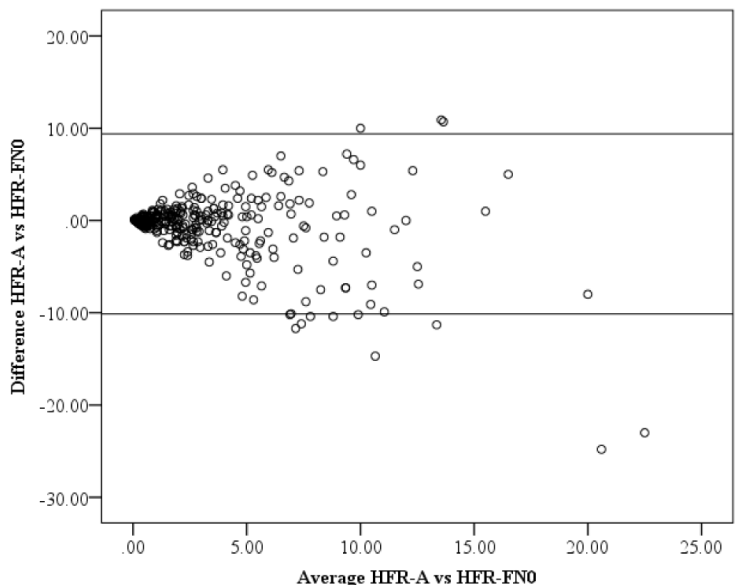

Figure 2. Bland Altman plot of HFR-A and HFR-FN

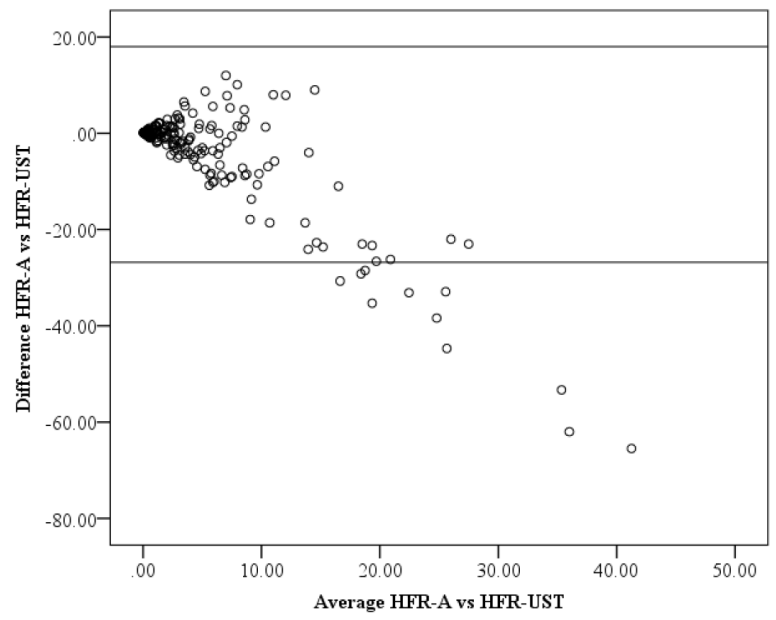

Figure 4. Bland Altman plot of HFR-A and HFR-UST. 


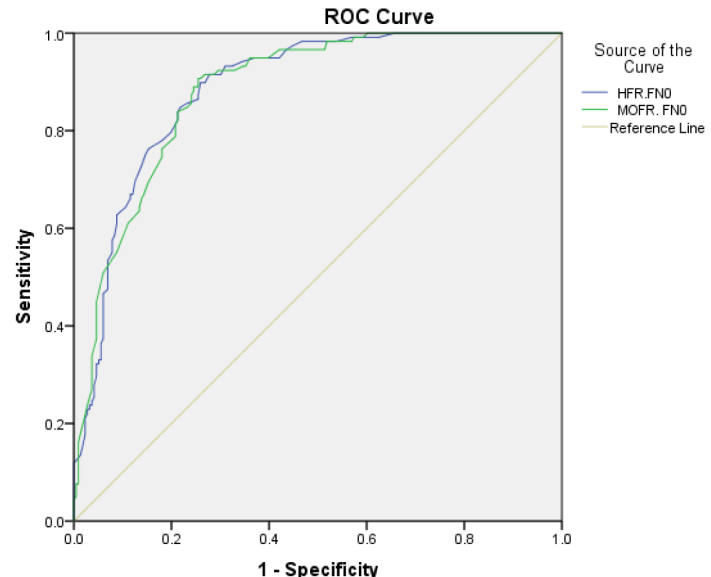

Figure 5. ROC curve for MOFR-FN $\mathrm{N}_{0}$ and HFR-FN

MOFR-FN - major osteoporotic fracture risk calculated without bone mineral density. HFR-FN - hip fracture risk calculated without bone mineral density.

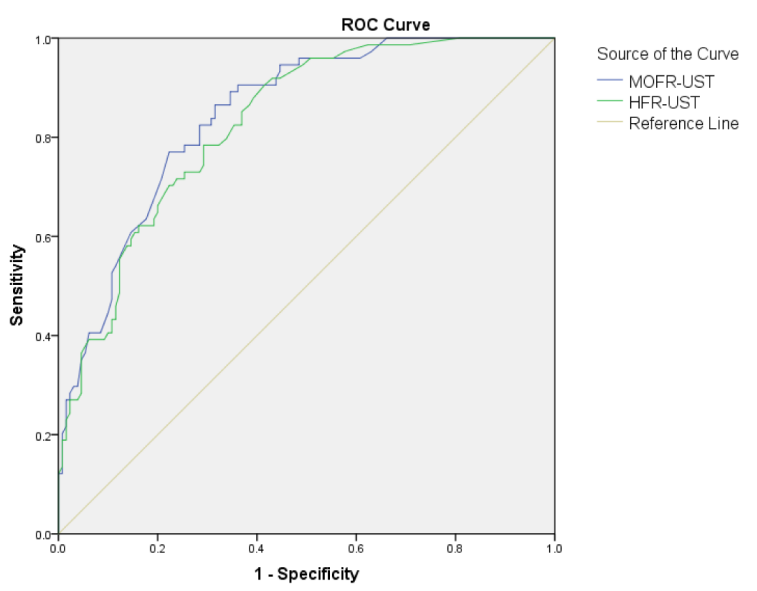

Figure 6. ROC curve for MOFR-UST and HFR-UST.

MOFR-UST - major osteoporotic fracture risk calculated with ultrasound T score. HFR-UST - hip fracture risk calculated with ultrasound T score.

The results of the regression analyses are shown in Table 4. Compared to fracture probabilities estimated with UST, fracture probabilities estimated without BMD showed greater $r$ and $\mathrm{R}^{2}$ and lesser measurement differences (SEE).

Table 4. Regression analysis comparing modified fracture probabilities against reference standard

\begin{tabular}{lcccc}
\hline Model & $r$ & $R^{2}$ & SEE & P value \\
\hline MOFR-FN $_{0}$ & 0.76 & 0.58 & 3.96 & $<0.001$ \\
HFR-FN $_{0}$ & 0.62 & 0.38 & 2.76 & $<0.001$ \\
MOFR-UST $_{\text {HFR-UST }}$ & 0.58 & 0.34 & 6.13 & $<0.001$ \\
\hline
\end{tabular}

$\mathrm{r}$ - correlation coefficient, $\mathrm{R}^{2}$ - coefficient of determination, SEE - standard error of estimate MOFR-FN - major osteoporotic fracture risk calculated without bone mineral density. HFR-FN calculated with ultrasound T score. HFR-UST - hip fracture risk calculated with ultrasound T score.

MOFR-FN ${ }_{0}$ and $\mathrm{HFR}-\mathrm{FN}_{0}$ showed strong correlations with age, body weight and $\mathrm{BMI}(\mathrm{p}<0.001$ for all associations, Table 5).

Table 5. Correlations of FRAX-FN ${ }_{0}$ and FRAX-UST with clinical risk factors

\begin{tabular}{lllclcc}
\hline & \multicolumn{2}{c}{ Age } & \multicolumn{3}{c}{ Bodyweight } & \multicolumn{2}{c}{ BMI } \\
\cline { 2 - 7 } & $r$ & $P$ value & $r$ & $P$ value & $r$ & $P$ value \\
\hline MOFR-A & 0.47 & $<0.001$ & -0.37 & $<0.001$ & -0.29 & $<0.001$ \\
HFR-A & 0.28 & $<0.001$ & -0.39 & $<0.001$ & -0.34 & $<0.001$ \\
MOFR-FN $_{0}$ & 0.68 & $<0.001$ & -0.39 & $<0.001$ & -0.32 & $<0.001$ \\
HFR-FN $_{0}$ & 0.60 & $<0.001$ & -0.45 & $<0.001$ & -0.42 & $<0.001$ \\
MOFR-UST & 0.35 & $<0.001$ & -0.27 & $<0.001$ & -0.22 & $<0.001$ \\
HFR-UST & 0.22 & $<0.001$ & -0.26 & $<0.001$ & -0.22 & $<0.001$ \\
\hline
\end{tabular}

$\mathrm{P}<0.001$ for all the associations. $\mathrm{r}$ - correlation coefficient, MOFR-A - Absolute major osteoporotic fracture risk. HFR-A - Absolute hip fracture risk. MOFR-FN - major osteoporotic fracture risk calculated without bone mineral density. HFR-FN ${ }_{0}$ - hip fracture risk calculated without bone mineral density. MOFR-UST - major osteoporotic fracture risk calculated with ultrasound T score. HFR-UST - hip fracture risk calculated with ultrasound $\mathrm{T}$ score. 


\section{Discussion}

Many countries have developed own FRAX models using national data on the assumption that countryspecific algorithms suit the local populations best. The accuracy of a particular FRAX model depends on the information that was used to build the model. Some countries such as Sri Lanka used data of a surrogate population to build the model.

This study compared Sri Lankan FRAX model with and without BMD data in predicting the 10-year probability of hip and major osteoporotic fractures among postmenopausal women. Furthermore, we assessed the validity of using US bone scan data (T-score of radius) instead of femoral neck BMD in SL-FRAX to predict fracture probabilities. Our data demonstrate that SL-FRAX without BMD input can be an alternative for clinicians working in places with no access to DXA facility. Inclusion of US T-score in the calculation did not improve the fracture risk predictability among this group of women.

When the femoral neck BMD was included in the calculation, $35.7 \%$ of the study participants had high fracture risk requiring pharmacological interventions. The SL-FRAX without BMD input slightly overestimated this value $(41 \%)$. However, the strong correlation between FRAX-FN ${ }_{0}$ and FRAX-A proves that fracture risk prediction by FRAX without BMD coincide with fracture risk predicted with BMD. In previous studies, noninclusion of BMD caused both overestimation and underestimation of the fracture risk [13-15]. FRAX without BMD has underdiagnosed both major osteoporosis and hip fracture risks in a Spanish women population. However, they did not use a FRAX tool specific for Spanish population in their study [13]. Egsmose et al. revealed that FRAX without BMD overestimated the fracture risk in a group of postmenopausal women who had suffered distal forearm fractures [14]. A similar observation was seen among patients with rheumatoid arthritis in the UK [15]. Gadam et al., however, found $84 \%$ identical fracture risk prediction by FRAX with and without BMD in a multiethnic study sample in the USA [11]. Further, Turkish postmenopausal women with osteopenia showed 93\% agreement between FRAX with and without BMD in assessing HFR [12]. A similar study in Canada showed that, Canadian FRAX tool without BMD is a good predictor of fracture risk in both men and women [26]. The evidence show that, there is a range of 76 to $90 \%$ agreement between FRAX scores determined with and without BMD [12].

Our data does not support the idea of substituting DXA derived BMD with QUS data in FRAX calculations. The FRAX-UST showed comparatively weak associations with FRAX-A. In Sri Lanka, QUS is used in areas where DXA is not readily available as it is relatively cheap and easy to use. QUS uses ultrasound waves to assess the bone quality and therefore the principles used in QUS are different from DXA. Furthermore, the utility of QUS in the field of osteoporosis is uncertain [27,28].

We observed a considerable overestimation of fracture risk by FRAX-UST ( $47.8 \%$ vs $35.7 \%$ ). The US bone scans are technologically different and the generated outcome largely depend on the site measured and the instrument used [29-31]. In addition the soft tissue layers covering the bone, its composition and thickness could interfere the precision of QUS measurements [32-35].

Both FRAX-FN ${ }_{0}$ and FRAX-UST had more than $80 \%$ AUC and showed their ability to discriminate high and low risk patients. Of them, FRAX-FN ${ }_{0}$ had greater AUC compared to FRAX-UST. The SEE produced by regression analysis is a measure of deviation of residuals from regression line with higher values indicating higher deviation. In our analysis, SEE of MOFR-FN ${ }_{0}$ and HFR$\mathrm{FN}_{0}$ showed a lesser variation when compared with similar values of QUS. In contrast, MOFR-UST and HFR-UST had comparatively higher SEE showing higher deviation from MOFR-A and HFR-A.

Age, body weight and BMI are predominant clinical risk factors of osteoporotic fractures. Probabilities of both MOFR and HFR calculated without BMD showed stronger correlations with age, body weight and BMI when compared with FRAX-UST. It implies that US SOS of the radius does not reflect the age-related changes in BMD. In support of this, studies have shown poor agreement between radial US and DXA technics and thus suggested radial QUS is not a good alternative to DXA $[27,31,36]$. On the other hand, QUS of calcaneus showed good correlations with DXA BMD $[28,37]$.

This study has some limitations. Since we have no electronic medical record system in Sri Lanka, we sometimes have to depend on the information provided by the patients about clinical risk factors to calculate FRAX. We, however, verified the information using personal health records. Further, the QUS scanning device we used could measure only the SOS and not the BUA. SOS and BUA together produce a parameter 'Stiffness Index' which more precisely describe the quality of the bone.

In conclusion, we suggest that Sri Lankan FRAX tool without BMD provides a reasonable alternative to assess fracture risk of postmenopausal women in places where DXA facility is not readily available. This will help to narrow down the current care gap in which many subjects with high fracture risk are not recognized and remain untreated. Radial QUS data cannot be used in FRAX in place of BMD to assess fracture risk.

\section{Acknowledgements}

Authors are thankful to the study participants and the staff of DEXA scanning unit, Teaching Hospital Karapitiya, Galle. Further we acknowledge the financial 
support by the postgraduate research grants of the University Grants Commission of Sri Lanka and the University of Wolverhampton, UK.

\section{Conflicts of interests}

The authors declare that there are no conflicts of interest.

\section{Declaration}

The authors declare that, this research paper was not published or not under consideration in part or as a whole in any other journal or a proceeding.

\section{References}

1. Choi EJ, Kim SA, Kim NR, Rhee JA, Yun YW, Shin MH. Risk Factors for Falls in Older Korean Adults: The 2011 Community Health Survey. J Korean Med Sci 2014; 29: 1482 .

2. Lee S, Choi MG, Yu J, et al. The effects of the Korean reference value on the prevalence of osteoporosis and the prediction of fracture risk. BMC Musculoskelet Disord 2015; 16: $1-13$.

3. International Osteoporosis Foundation. Facts and statistics [Internet]. IOF international. 2017 [cited 2018 Jul 17]. Available from: https://www.iofbonehealth.org/factsstatistics\#category-14

4. Ettinger B, Black DM, Dawson-Hughes B, Pressman AR, Melton LJ. Updated fracture incidence rates for the US version of FRAX®. Osteoporos Int 2010; 21: 25-33.

5. Burge R, Dawson-Hughes B, Solomon DH, Wong JB, King A, Tosteson A. Incidence and economic burden of osteoporosis-related fractures in the United States, 20052025. J Bone Miner Res 2007; 22: 465-75.

6. Cooper C, Campion G, Melton LJ. Hip fractures in the elderly: A world-wide projection. Osteoporos Int 1992; 2: 285-9.

7. Gullberg B, Johnell O, Kanis JA. World-wide projections for hip fracture. Osteoporos Int 1997; 7: 407-13.

8. Cheung EYN, Tan KCB, Cheung C-L, Kung AWC. Osteoporosis in East Asia: Current issues in assessment and management. Osteoporos Sarcopenia 2016; 2: 118-33.

9. Kanis JA, McCloskey EV, Johansson H, Oden A, Ström O, Borgström F. Development and use of FRAX ${ }^{\circledR}$ in osteoporosis. Osteoporos Int 2010; 21: 407-13.

10. Shenoy S, Madduri S, Cherqaoui R, Nunlee-Bland G. 10year frax calculations with and without femoral neck bone mineral density in African American patients with type 2 diabetes. Endocr Rev 2014; 35.

11. Gadam RK, Schlauch K, Izuora KE. Frax Prediction without BMD for Assessment of Osteoporotic Fracture Risk. Endocr Pract 2013; 19: 780-4.
12. Sarikaya NO, Yavasi SK, Tan G, et al. Agreement between FRAX scores calculated with and without bone mineral density in women with osteopenia in Turkey. Clin Rheumatol 2014; 33: 1785-9.

13. Azagra R, Roca G, Encabo G, et al. FRAX® tool, the WHO algorithm to predict osteoporotic fractures: The first analysis of its discriminative and predictive ability in the Spanish FRIDEX cohort. BMC Musculoskelet Disord 2012; 13.

14. Egsmose EL, Birkvig M, Buhl T, Madsen OR. FRAX fracture risk in women with a recent fracture of the distal forearm: agreement between assessments with and without bone mineral density and impact of measurement side in the individual patient. Clin Rheumatol 2015; 34: 1265-72.

15. Klop C, De Vries F, Bijlsma JWJ, Leufkens HGM, Welsing PMJ. Predicting the 10-year risk of hip and major osteoporotic fracture in rheumatoid arthritis and in the general population: An independent validation and update of UK FRAX without bone mineral density. Ann Rheum Dis 2016; 75: 2095-100.

16. Alquaiz AM, Kazi A, Tayel S, et al. Prevalence and factors associated with low bone mineral density in Saudi women: A community based survey. BMC Musculoskelet Disord $2014 ; 15$.

17. Kanis JA, McCloskey EV, Johansson H, Oden A, Melton LJ, Khaltaev N. A reference standard for the description of osteoporosis. Bone 2008; 42: 467-75.

18. Naves M, Díaz-López JB, Gómez C, Rodríguez-Rebollar A, Serrano-Arias M, Cannata-Andía JB. Prevalence of osteoporosis in men and determinants of changes in bone mass in a non-selected Spanish population. Osteoporos Int 2005; 16: 603-9.

19. Nayak S, Olkin I, Liu H, Grabe M, Gould MK, Allen IE. Annals of Internal Medicine Review Meta-Analysis?: Accuracy of Quantitative Ultrasound for Identifying Patients with Osteoporosis. Ann Intern Med 2006; 144: 832-41.

20. Siribaddana SH, Kovas Y, Fernando DJS. Quantitative ultrasound of bone and calcium intake in suburban males in Sri Lanka. Int J Rheum Dis 2008; 11: 407-13.

21. Marín F, González-Macías J, Díez-Pérez A, Palma S, Delgado-Rodríguez M. Relationship between bone quantitative ultrasound and fractures: A meta-analysis. $J$ Bone Miner Res 2006; 21: 1126-35.

22. McCloskey EV, Kanis JA, Odén A, et al. Predictive ability of heel quantitative ultrasound for incident fractures: an individual-level meta-analysis. Osteoporos Int 2015; 26: 1979-87.

23. Kanis JA, McCloskey EV, Harvey NC, Johansson H, Leslie WD. Intervention thresholds and the diagnosis of osteoporosis. J Bone Miner Res 2015; 30: 1747-53.

24. Kanis JA, Melton LJ, Christiansen C, Johnston CC, Khaltaev N. The diagnosis of osteoporosis. J Bone Miner Res 1994; 9: 1137-41. 
25. Lekamwasam S. Sri Lankan FRAX model and countryspecific intervention thresholds. Arch Osteoporos 2013; 8.

26. Fraser LA, Langsetmo L, Berger C, et al. Fracture prediction and calibration of a Canadian FRAX ${ }^{\circledR}$ tool: A populationbased report from CaMos. Osteoporos Int 2011; 22: 829-37.

27. Williams JE, Wilson CM, Biassoni L, Suri R, Fewtrell MS. Dual energy $\mathrm{x}$-ray absorptiometry and quantitative ultrasound are not interchangeable in diagnosing abnormal bones. Arch Dis Child 2012; 97: 822-4.

28. Hashmi FR, Elfandi KO. Heel ultrasound scan in detecting osteoporosis in low trauma fracture patients. Orthop Rev 2016; 8: 61-3.

29. Hans D, Krieg MA. Quantitative ultrasound for the detection and management of osteoporosis. Salud Publica Mex 2009; 51: S25-37.

30. Krieg MA, Barkmann R, Gonnelli S, et al. Quantitative Ultrasound in the Management of Osteoporosis: The 2007 ISCD Official Positions. J Clin Densitom 2008; 11: 163-87.

31. Chong KH, Poh BK, Jamil NA, Kamaruddin NA, Deurenberg P. Radial quantitative ultrasound and dual energy $\mathrm{X}$-ray absorptiometry: Intermethod agreement for bone status assessment in children. Biomed Res Int 2015; 2015.
32. Malo MKH, Karjalainen JP, Isaksson H, Riekkinen O, Jurvelin JS, Töyräs J. Numerical Analysis of Uncertainties in Dual Frequency Bone Ultrasound Technique. Ultrasound Med Biol 2010; 36: 288-94.

33. Riekkinen O, Hakulinen MA, Timonen M, Töyräs J, Jurvelin JS. Influence of overlying soft tissues on trabecular bone acoustic measurement at various ultrasound frequencies. Ultrasound Med Biol 2006; 32: 1073-83.

34. Stein EM, Rosete F, Young P, et al. Clinical assessment of the $1 / 3$ radius using a new desktop ultrasonic bone densitometer. Ultrasound Med Biol 2014; 8: 1385-95.

35. Eliakim A, Nemet D, Wolach B. Quantitative ultrasound measurements of bone strength in obese children and adolescents. J Pediatr Endocrinol Metab 2001; 14: 159-64.

36. Khan KM, Sarafoglou K, Somani A, Frohnert B, Miller BS. Can ultrasound be used to estimate bone mineral density in children with growth problems? Acta Paediatr Int J Paediatr $2013 ; 102$.

37. Rajendran K, Suthakaran PK, Devayani L, et al. Evaluation of osteoporosis using calcaneal QUS and FRAX score as a screening tool in a semi urban tertiary care hospital of South India. Int J Adv Med 2015; 2: 341-5. 